\title{
一类混合分红策略下的广义 $\operatorname{Erlang}(n)$ 风险模型
}

\author{
温玉珍*，尹传存
}

曲阜师范大学数学科学学院, 曲阜 273165

E-mail: wenyzhen@163.com, ccyin@mail.qfnu.edu.cn

收稿日期: 2014-03-06; 接受日期: 2014-06-03; * 通信作者

国家自然科学基金 (批准号: 11171179 和 11301295) 和中国高等学校博士点科研基金 (批准号: 20133705110002) 资助项目

摘要 本文考虑混合分红策略下索赔来到间隔为广义 $\operatorname{Erlang}(n)$ 分布的更新风险模型, 利用指数分布 的无记忆性, 分别得到破产前期望折现分红函数和折现分红的矩母函数满足的积分 - 微分方程及其边 界条件. 最后给出索赔为指数分布及索赔来到间隔为广义 $\operatorname{Erlang}(2)$ 分布的风险模型的期望折现分红 函数的精确表达式.

关键词 混合分红策略 折现分红函数 广义 $\operatorname{Erlang}(n)$ 分布

MSC (2010) 主题分类 $62 \mathrm{P} 05,62 \mathrm{~K} 05$

\section{1 引言}

近年来, 分红问题在精算界受到了广泛的关注. 在对分红问题的研究中, 障碍分红策略和阈值分 红策略被广泛研究. 在障碍分红策略中, 最常见的是常数值障碍分红策略. 这种分红策略是指当盈余 不超过分红界时不分红; 当盈余超过分红界时, 超出部分全部进行分红. 对常数值障碍分红策略的研 究最早可追溯到 De Finetti (1957). De Finetti 首次研究了索赔额为 \pm 1 的随机游动的障碍分红问题. 后来许多学者又研究了不同风险模型下的障碍分红问题, 例如, Lin 等人 ${ }^{[1]}$ 研究了障碍分红策略下的 Gerber-Shiu 函数; Yuen 和 Yin ${ }^{[2]}$ 研究了障碍分红策略下 Lévy 风险过程的最优分红问题. 障碍分红 策略常用来描述分红率不受限制的分红策略, 然而, 如果采用障碍分红策略, 则公司一定破产, 这在很 多情形下是不理想的. 后来, Jeanblanc-Picqué和 Shiryaev ${ }^{[3]}$ 以及 Asmussen 和 Taksar ${ }^{[4]}$ 对分红加以 限制, 提出了修正后的障碍分红策略, 即阈值分红策略. 阈值分红策略是指当盈余不超过分红界时不分 红; 当盈余超过分红界时, 超出部分以常数值分红率进行分红. 许多学者在经典的保险风险模型中得 到了此分红策略下的有趣结果, 例如, $\mathrm{Ng}{ }^{[5]}$ 考虑了阈值分红策略下的复合 Poisson 模型的对偶模型, 并得到了期望折现分红的两个积分 - 微分方程, 当上跳是指数分布或混合指数分布时得到了期望折现 分红的精确表达式, 并对一般的上跳分布利用 Laplace 变换对期望折现分红进行了讨论. Wan ${ }^{[6]}$ 考虑 了阈值分红策略下的带扰动的复合 Poisson 模型, 得到了期望折现分红的精确表达式. 其他对阈值分 红策略的研究可参见文献 [7-13].

$\mathrm{Ng}$ [14] 考虑了混合分红策略下索赔额为相位分布的对偶风险模型, 并得到了期望折现分红和破产 时刻的 Laplace 变换的精确表达式. 混合分红策略是指存在两个正常数 $b_{1}$ 和 $b_{2}\left(b_{2}>b_{1}>0\right)$, 当公 司盈余低于 $b_{1}$ 时不分红; 当公司盈余在区域 $\left(b_{1}, b_{2}\right)$ 时以常数值分红率进行分红; 当公司盈余超过 $b_{2}$ 
时, 盈余全部进行分红. 上述混合分红策略可以看作一个单一的障碍分红策略和单一的阈值分红策略 的推广. 显然, 混合分红策略比一个单一的障碍分红策略更贴近现实. 对于一个公司来说, 这种分红策 略在理论上比一个公司采取一个要么不分红要么全部分红的策略更加灵活. 同样, 这种分红策略也比 阈值分红策略更加贴近现实, 因为保险公司的盈余过程不可能无限的增长.

我们可以利用同样的方法考虑带反射壁的多层分红问题, 也就是保险公司按照修正盈余的 $n+1$ 个 层次来分发红利, 令 $0=b_{0}<b_{1}<\cdots<b_{n}<\infty$, 当修正的盈余过程在 $b_{i-1}$ 与 $b_{i}$ 之间时, 保费率是 $c_{i}$, 其中 $i=1,2, \ldots, n$; 而当修正的盈余过程超过水平 $b_{n}$ 时将全部分红. 本文考虑的是这种带反射壁的 多层分红策略的特殊情形, 即 $n=2$ 时的特殊情形.

受文献 [14] 的影响, 本文继续考虑混合分红策略下的索赔间隔为广义 $\operatorname{Erlang}(n)$ 分布的更新风险 模型. 本文结构如下: 第 2 节首先介绍风险模型; 第 3 节得到期望折现分红函数和折现分红的矩母函 数满足的积分 - 微分方程及边界条件.

\section{2 模型简介}

在经典的风险模型 $[15]$ 中, 保险公司在时刻 $t$ 的盈余为

$$
U(t)=u+c t-\sum_{k=1}^{N(t)} Z_{k} \equiv u+c t-S(t), \quad t \geqslant 0,
$$

其中 $u$ 和 $c$ 是常数; $u \geqslant 0$ 是初始盈余; $c>0$ 是保费率; $S(t)=\sum_{k=1}^{N(t)} Z_{k}$ 是到时刻 $t$ 的累计索赔额; $\left\{Z_{k}\right\}_{k \geqslant 1}$ 是一列取非负值的独立同分布的随机变量, 其分布函数为 $P(x)$, 密度函数为 $p(x) ;\{N(t)\}$ 是 强度为 $\lambda$ 的 Poisson 过程, 并且与 $\left\{Z_{k}\right\}_{k \geqslant 1}$ 独立. 在模型 (1.1) 中, 单位时间的期望增长盈余为

$$
\mu=c-E[S(1)]=c-\lambda \int_{0}^{+\infty} x p(x) d x .
$$

本文假设 $\mu>0$.

在本文中, 我们假设到时刻 $t$ 的索赔计数过程是如下的更新过程:

$$
N(t)=\max \left\{k \geqslant 1: W_{1}+W_{2}+\cdots+W_{k} \leqslant t\right\},
$$

其中随机变量 $\left\{W_{i}\right\}_{i \geqslant 1}$ 是独立的且均为广义 $\operatorname{Erlang}(n)$ 分布的随机变量, 即随机变量 $W_{i}$ 是 $n$ 个独立 的指数分布的和:

$$
W_{i}=\xi_{i 1}+\xi_{i 2}+\cdots+\xi_{\text {in }}, \quad i=1,2, \ldots,
$$

其中 $\xi_{i j}(j=1,2, \ldots, n)$ 是参数为 $\lambda_{j}>0$ 的指数分布的随机变量.

我们假设保险公司按照混合分红策略进行分红, 两个分红界分别是 $b_{1}$ 和 $b_{2}\left(b_{2}>b_{1}>0\right)$. 在这 种分红策略下红利由两部分组成, 第一部分红利是指当公司的修正盈余过程的盈余在 $b_{1}$ 与 $b_{2}$ 之间 时, 按照常值分红率 $\alpha$ 进行分红而得; 第二部分红利是指当公司的修正盈余过程超过水平 $b_{2}$ 时, 超出 部分全部用来分红所得. 当 $t>0$ 时, 令 $D(t)=D_{1}(t)+D_{2}(t)$ 表示到时刻 $t$ 的累积分红, 其中 $D_{1}(t)$ 和 $D_{2}(t)$ 分别表示我们在上面提到的两部分红利. 定义 $X(t)=U(t)-D(t), t \geqslant 0$ 是混合分红策略下 公司在时刻 $t$ 的修正盈余. 令 $T=\inf \{t \geqslant 0: X(t)<0\}$ 是修正盈余过程 $X$ 的破产时刻. 令

$$
D=\int_{0}^{T} \mathrm{e}^{-\delta t} d D(t)=D_{1}+D_{2}
$$


是破产前的累积折现分红, 其中 $\delta>0$ 是折现率,

$$
D_{1}=\int_{0}^{T} \mathrm{e}^{-\delta t} d D_{1}(t)=\int_{0}^{T} \alpha \mathrm{e}^{-\delta t} I_{\left\{\left(b_{1}<X(t) \leqslant b_{2}\right\}\right.} d t, \quad D_{2}=\int_{0}^{T} \mathrm{e}^{-\delta t} d D_{2}(t),
$$

这里 $D_{2}(t)=\max _{0<\tau<t}\left(U(\tau)-D_{1}(\tau)\right)-b_{2}$.

\section{3 主要结果}

本节考虑混合分红策略下的期望折现分红函数. 记 $V\left(u ; b_{1}, b_{2}\right), V_{d}\left(u ; b_{1}, b_{2}\right)$ 和 $V_{r}\left(u ; b_{1}, b_{2}\right)$ 分别 是 $D, D_{1}$ 和 $D_{2}$ 的期望, 即

$$
\begin{aligned}
& V\left(u ; b_{1}, b_{2}\right)=E[D \mid X(0)=u] \equiv E_{u}[D], \\
& V_{d}\left(u ; b_{1}, b_{2}\right)=E\left[D_{1} \mid X(0)=u\right] \equiv E_{u}\left[D_{1}\right], \\
& V_{r}\left(u ; b_{1}, b_{2}\right)=E\left[D_{2} \mid X(0)=u\right] \equiv E_{u}\left[D_{2}\right] .
\end{aligned}
$$

我们记

$$
V_{d}\left(u ; b_{1}, b_{2}\right)=\left\{\begin{array}{ll}
V_{d 1}\left(u ; b_{1}, b_{2}\right), & 0 \leqslant u<b_{1}, \\
V_{d 2}\left(u ; b_{1}, b_{2}\right), & b_{1} \leqslant u \leqslant b_{2},
\end{array} \quad V_{r}\left(u ; b_{1}, b_{2}\right)= \begin{cases}V_{r 1}\left(u ; b_{1}, b_{2}\right), & 0 \leqslant u<b_{1}, \\
V_{r 2}\left(u ; b_{1}, b_{2}\right), & b_{1} \leqslant u \leqslant b_{2} .\end{cases}\right.
$$

于是, 我们可得

$$
V\left(u ; b_{1}, b_{2}\right)= \begin{cases}V_{1 *}\left(u ; b_{1}, b_{2}\right)=V_{d 1}\left(u ; b_{1}, b_{2}\right)+V_{r 1}\left(u ; b_{1}, b_{2}\right), & 0 \leqslant u<b_{1}, \\ V_{2 *}\left(u ; b_{1}, b_{2}\right)=V_{d 2}\left(u ; b_{1}, b_{2}\right)+V_{r 2}\left(u ; b_{1}, b_{2}\right), & b_{1} \leqslant u \leqslant b_{2} .\end{cases}
$$

注意到当 $u>b_{2}$ 时, 我们有

$$
V\left(u ; b_{1}, b_{2}\right)=u-b_{2}+V\left(b_{2} ; b_{1}, b_{2}\right) .
$$

下面先推导期望折现分红函数 $V_{d}\left(u ; b_{1}, b_{2}\right)$ 和 $V_{r}\left(u ; b_{1}, b_{2}\right)$ 满足的积分 - 微分方程及其边界条件.

记 $\frac{\partial}{\partial u}$ 表示关于 $u$ 的微分算子. 对任意的 $k \in \mathbb{N}^{+}$, 我们定义 $\prod_{j=k}^{k-1} \cdot=1$ 和 $\sum_{j=k}^{k-1} \cdot=0$. 为叙述方 便, 我们把 $V\left(u ; b_{1}, b_{2}\right), V_{d}\left(u ; b_{1}, b_{2}\right), V_{r}\left(u ; b_{1}, b_{2}\right), V_{1 *}\left(u ; b_{1}, b_{2}\right), V_{2 *}\left(u ; b_{1}, b_{2}\right), V_{d 1}\left(u ; b_{1}, b_{2}\right), V_{d 2}\left(u ; b_{1}, b_{2}\right)$, $V_{r 1}\left(u ; b_{1}, b_{2}\right)$ 和 $V_{r 2}\left(u ; b_{1}, b_{2}\right)$ 分别简记为 $V(u), V_{d}(u), V_{r}(u), V_{1 *}(u), V_{2 *}(u), V_{d 1}(u), V_{d 2}(u), V_{r 1}(u)$ 和 $V_{r 2}(u)$.

定理 $1 \quad V_{d}(u)$ 满足下面的积分 - 微分方程:

（1）当 $0<u<b_{1}$ 时, 有

$$
\left[\prod_{j=1}^{n}\left(1+\frac{\delta}{\lambda_{j}}-\frac{c}{\lambda_{j}} \frac{\partial}{\partial u}\right)\right] V_{d 1}(u)=\int_{0}^{u} V_{d 1}(u-x) p(x) d x
$$

（2）当 $b_{1}<u<b_{2}$ 时, 有

$$
\begin{gathered}
{\left[\prod_{j=1}^{n}\left(1+\frac{\delta}{\lambda_{j}}-\frac{c-\alpha}{\lambda_{j}} \frac{\partial}{\partial u}\right)\right] V_{d 2}(u)-\sum_{j=1}^{n}\left(\prod_{i=j+1}^{n} \frac{\lambda_{i}+\delta}{\lambda_{i}}\right) \frac{\alpha}{\lambda_{j}}} \\
\quad=\int_{0}^{u-b_{1}} V_{d 2}(u-x) p(x) d x+\int_{u-b_{1}}^{u} V_{d 1}(u-x) p(x) d x
\end{gathered}
$$


边界条件是

$$
\begin{aligned}
{\left.\left[\prod_{j=1}^{k}\left(1+\frac{\delta}{\lambda_{j}}-\frac{c-\alpha}{\lambda_{j}} \frac{\partial}{\partial u}\right)\right] \frac{\partial V_{d 2}(u)}{\partial u}\right|_{u=b_{2}-} } & =0, \quad k=0,1,2, \ldots, n-1 \\
{\left.\left[\prod_{j=1}^{k}\left(1+\frac{\delta}{\lambda_{j}}-\frac{c}{\lambda_{j}} \frac{\partial}{\partial u}\right)\right] V_{d 1}(u)\right|_{u=b_{1}-}=} & {\left.\left[\prod_{j=1}^{k}\left(1+\frac{\delta}{\lambda_{j}}-\frac{c-\alpha}{\lambda_{j}} \frac{\partial}{\partial u}\right)\right] V_{d 2}(u)\right|_{u=b_{1}+} } \\
& -\sum_{j=1}^{k}\left(\prod_{i=j+1}^{k} \frac{\lambda_{i}+\delta}{\lambda_{i}}\right) \frac{\alpha}{\lambda_{j}}, \quad k=0,1,2, \ldots, n-1 .
\end{aligned}
$$

证明 当 $0<u<b_{1}$ 时, 利用 $\operatorname{Erlang}(n)$ 分布是 $n$ 个独立指数分布的和这一性质, 仿照文献 [16], 我们令 $V_{d 1 j}(u)$ 是风险过程在状态 $j(j=1, \ldots, n)$ 时的期望折现分红函数, 显然有 $V_{d 1}(u)=V_{d 11}(u)$. 考虑在无穷小时间区间 $[0, d t]$ 内是否有索赔发生, 当 $0 \leqslant u<b_{1}$ 且 $j=1, \ldots, n-1$ 时, 我们有

$$
V_{d 1 j}(u)=\mathrm{e}^{-\delta d t}\left\{P\left(\xi_{j}>d t\right) V_{d 1 j}(u+c d t)+P\left(\xi_{j} \leqslant d t\right) V_{d 1 j+1}(u+c d t)\right\} .
$$

又

$$
\begin{aligned}
& \mathrm{e}^{-\delta d t}=1-\delta d t+o(d t), \\
& P\left(\xi_{j}>d t\right)=1-\lambda_{j} d t+o(d t), \\
& P\left(\xi_{j} \leqslant d t\right)=\lambda_{j} d t+o(d t), \\
& V_{d 1 j}(u+c d t)=V_{d 1 j}(u)+c \frac{\partial V_{d 1 j}(u)}{\partial u} d t+o(d t) .
\end{aligned}
$$

把上式带入到 (3.5) 中, 计算后可得

$$
\lambda_{j} V_{d 1 j+1}(u)=\left[\left(\lambda_{j}+\delta\right)-c \frac{\partial}{\partial u}\right] V_{d 1 j}(u) .
$$

当 $j=n$ 时, 我们有

$$
\begin{aligned}
V_{d 1 n}(u) & =\mathrm{e}^{-\delta d t}\left[P\left(\xi_{n}>d t\right) V_{d 1 n}(u+c d t)+P\left(\xi_{n} \leqslant d t\right) \int_{0}^{u} V_{d 1}(u-x) p(x) d x\right]+o(d t) \\
& =V_{d 1 n}(u)-\left(\delta+\lambda_{n}\right) V_{d 1 n}(u) d t+c \frac{\partial V_{d 1 n}(u)}{\partial u} d t+\lambda_{n} d t \int_{0}^{u} V_{d 1}(u-x) p(x) d x+o(d t),
\end{aligned}
$$

整理得

$$
\left[\left(\lambda_{n}+\delta\right)-c \frac{\partial}{\partial u}\right] V_{d 1 n}(u)=\lambda_{n} \int_{0}^{u} V_{d 1}(u-x) p(x) d x
$$

利用 (3.6) 可得

$$
V_{d 1 j+1}(u)=\left[\frac{\left(\lambda_{j}+\delta\right)-c \frac{\partial}{\partial u}}{\lambda_{j}}\right] V_{d 1 j}(u), \quad j=1,2, \ldots, n-1,
$$

故有

$$
V_{d 1 n}(u)=\left[\prod_{j=1}^{n-1} \frac{\left(\lambda_{j}+\delta\right)-c \frac{\partial}{\partial u}}{\lambda_{j}}\right] V_{d 1}(u) .
$$


结合 (3.7) 即得结论 (3.1).

(2) 当 $b_{1}<u<b_{2}$ 时, 我们有

$$
V_{d 2 j}(u)=\alpha t \mathrm{e}^{-\delta d t}+\mathrm{e}^{-\delta d t}\left\{P\left(\xi_{j}>d t\right) V_{d 2 j}(u+(c-\alpha) d t)+P\left(\xi_{j} \leqslant d t\right) V_{d 2 j+1}(u+(c-\alpha) d t)\right\},
$$

计算后得

$$
\lambda_{j} V_{d 2 j+1}(u)=\left[\left(\lambda_{j}+\delta\right)-(c-\alpha) \frac{\partial}{\partial u}\right] V_{d 2 j}(u)-\alpha .
$$

当 $j=n$ 时, 同理可得

$$
\begin{aligned}
V_{d 2 n}(u)=\alpha & +\mathrm{e}^{-\delta d t} P\left(\xi_{n}>d t\right) V_{d 2 n}(u+(c-\alpha) d t)+o(d t)+\mathrm{e}^{-\delta d t} P\left(\xi_{n} \leqslant d t\right) \\
& \left.\times\left[\int_{0}^{u-b_{1}} V_{d 2}(u+(c-\alpha) d t-x) p(x) d x+\int_{u-b_{1}}^{u} V_{d 1}(u+(c-\alpha) d t)-x\right) p(x) d x\right] \\
=\alpha & +V_{d 2 n}(u)-\left(\delta+\lambda_{n}\right) V_{d 2 n}(u) d t+(c-\alpha) \frac{\partial V_{d 2 n}(u)}{\partial u} d t \\
+ & {\left[\int_{0}^{u-b_{1}} V_{d 2}(u-x) p(x) d x+\int_{u-b_{1}}^{u} V_{d 1}(u-x) p(x) d x\right] \lambda_{n} d t+o(d t) . }
\end{aligned}
$$

结合 (3.10) 即得结论 (3.2).

下面证明边界条件 (3.3). 当 $u=b_{2}$ 且 $j=1,2, \ldots, n-1$ 时, 类似可得

$$
V_{d 2 j}\left(b_{2}\right)=\alpha d t+\mathrm{e}^{-\delta d t}\left\{P\left(\xi_{j}>d t\right) V_{d 2 j}\left(b_{2}\right)+P\left(\xi_{j} \leqslant d t\right) V_{d 2 j+1}\left(b_{2}\right)\right\} .
$$

利用 Taylor 展式有

$$
\lambda_{j} V_{d 2 j+1}\left(b_{2}\right)=\left(\lambda_{j}+\delta\right) V_{d 2 j}\left(b_{2}\right)-\alpha .
$$

令 $u \uparrow b_{2}$ 且与 $(3.10)$ 作比较可得

$$
\left.\frac{\partial V_{d 2 j}(u)}{\partial u}\right|_{u=b_{2}-}=0, \quad j=1,2, \ldots, n-1 .
$$

同样可验证 (3.12) 在 $j=n$ 时也成立. 利用 (3.10) 得

$$
V_{d 2 k}(u)=\left[\prod_{j=1}^{k} \frac{\left(\lambda_{j}+\delta\right)-(c-\alpha) \frac{\partial}{\partial u}}{\lambda_{j}}\right] V_{d 2}(u)-\sum_{j=1}^{k}\left(\prod_{i=j+1}^{k} \frac{\lambda_{i}+\delta}{\lambda_{i}}\right) \frac{\alpha}{\lambda_{j}}, \quad k=0,1,2, \ldots, n-1 .
$$

在上式两边同时作用算子 $\frac{\partial}{\partial u}$ 得

$$
\left.\left[\prod_{j=1}^{k} \frac{\left(\lambda_{j}+\delta\right)-(c-\alpha) \frac{\partial}{\partial u}}{\lambda_{j}}\right] \frac{\partial V_{d 2}(u)}{\partial u}\right|_{u=b_{2}-}=\left.\frac{\partial V_{d 2 k}(u)}{\partial u}\right|_{u=b_{2}-}=0, \quad k=0,1,2, \ldots, n-1,
$$

上式即是边界条件 (3.3). 同理, 利用 (3.8)、(3.10) 和 $V_{d 1}\left(b_{1}\right)=V_{d 2}\left(b_{1}\right)$ 可得边界条件 (3.4). 定理 得证.

注 1 在定理 1 中, 令 $b_{2} \rightarrow \infty$, 且索赔来到间隔 $W_{i}$ 是参数为 $\lambda$ 的指数分布, 即 $k(y)=\lambda \mathrm{e}^{-\lambda y}$, $y \geqslant 0$, 其中 $\lambda>0$, 则 (3.1) 和 (3.2) 分别是文献 [17] 中的 (5.1) 和 (5.2). 
定理 $2 V_{r}(u)$ 满足下面的积分 - 微分方程:

(1) 当 $0<u<b_{1}$ 时, 有

$$
\left[\prod_{j=1}^{n}\left(1+\frac{\delta}{\lambda_{j}}-\frac{c}{\lambda_{j}} \frac{\partial}{\partial u}\right)\right] V_{r 1}(u)=\int_{0}^{u} V_{r 1}(u-x) p(x) d x
$$

(2) 当 $b_{1}<u<b_{2}$ 时, 有

$$
\left[\prod_{j=1}^{n}\left(1+\frac{\delta}{\lambda_{j}}-\frac{c-\alpha}{\lambda_{j}} \frac{\partial}{\partial u}\right)\right] V_{r 2}(u)=\int_{0}^{u-b_{1}} V_{r 2}(u-x) p(x) d x+\int_{u-b_{1}}^{u} V_{r 1}(u-x) p(x) d x,
$$

当 $k=0,1,2, \ldots, n-1$ 时, 边界条件是

$$
\begin{aligned}
& {\left.\left[\prod_{j=1}^{k}\left(1+\frac{\delta}{\lambda_{j}}-\frac{c-\alpha}{\lambda_{j}} \frac{\partial}{\partial u}\right)\right] \frac{\partial V_{r 2}(u)}{\partial u}\right|_{u=b_{2}-}=1} \\
& {\left.\left[\prod_{j=1}^{k}\left(1+\frac{\delta}{\lambda_{j}}-\frac{c}{\lambda_{j}} \frac{\partial}{\partial u}\right)\right] V_{r 1}(u)\right|_{u=b_{1}-}=\left.\left[\prod_{j=1}^{k}\left(1+\frac{\delta}{\lambda_{j}}-\frac{c-\alpha}{\lambda_{j}} \frac{\partial}{\partial u}\right)\right] V_{r 2}(u)\right|_{u=b_{1}+} .}
\end{aligned}
$$

证明 (3.13)、(3.14) 和 (3.16) 的证明同 (3.1)、(3.2) 和 (3.4) 的证明类似, 此处我们省去证明. 当 $u=b_{2}$ 时, 对 $j=1,2, \ldots, n-1$, 仿照定理 3.1 的证明类似可得

$$
V_{r 2 j}\left(b_{2}\right)=\mathrm{e}^{-\delta d t}\left\{P\left(\xi_{j}>d t\right)\left(V_{r 2 j}\left(b_{2}\right)+(c-\alpha) t\right)+P\left(\xi_{j} \leqslant d t\right) V_{r 2 j+1}\left(b_{2}\right)\right\} .
$$

利用 Taylor 展式可得

$$
\lambda_{j} V_{r 2 j+1}\left(b_{2}\right)=\left(\lambda_{j}+\delta\right) V_{d 2 j}\left(b_{2}\right)-(c-\alpha)
$$

在等式 $V_{r 2 j+1}(u)=\left[\frac{\left(\lambda_{j}+\delta\right)-(c-\alpha) \frac{\partial}{\partial u}}{\lambda_{j}}\right] V_{r 2 j}(u)$ 中, 令 $u \uparrow b_{2}$ 且比较二等式可得

$$
\left.\frac{\partial V_{r 2 j}(u)}{\partial u}\right|_{u=b_{2}-}=1, \quad j=1,2, \ldots, n-1 .
$$

同样可以验证当 $j=n$ 时, (3.18) 也成立. 又由 (3.18) 得

$$
V_{d 2 k}(u)=\left[\prod_{j=1}^{k} \frac{\left(\lambda_{j}+\delta\right)-(c-\alpha) \frac{\partial}{\partial u}}{\lambda_{j}}\right] V_{d 2}(u), \quad k=0,1,2, \ldots, n-1 .
$$

上式两端同时作用算子 $\frac{\partial}{\partial u}$, 有

$$
\left.\left[\prod_{j=1}^{k} \frac{\left(\lambda_{j}+\delta\right)-(c-\alpha) \frac{\partial}{\partial u}}{\lambda_{j}}\right] \frac{\partial V_{d 2}(u)}{\partial u}\right|_{u=b_{2}-}=\left.\frac{\partial V_{d 2 k}(u)}{\partial u}\right|_{u=b_{2}-}=0, \quad k=0,1,2, \ldots, n-1,
$$

此即边界条件 (3.15). 定理得证.

根据 $V(u)$ 的定义及定理 1 和 2 可得 $V(u)$ 满足的积分 - 微分方程及边界条件.

定理 $3 \quad V(u)$ 满足下面的积分 - 微分方程: 
(1) 当 $0<u<b_{1}$ 时, 有

$$
\left[\prod_{j=1}^{n}\left(1+\frac{\delta}{\lambda_{j}}-\frac{c}{\lambda_{j}} \frac{\partial}{\partial u}\right)\right] V_{1 *}(u)=\int_{0}^{u} V_{1 *}(u-x) p(x) d x
$$

(2) 当 $b_{1}<u<b_{2}$ 时, 有

$$
\begin{gathered}
{\left[\prod_{j=1}^{n}\left(1+\frac{\delta}{\lambda_{j}}-\frac{c-\alpha}{\lambda_{j}} \frac{\partial}{\partial u}\right)\right] V_{2 *}(u)-\sum_{j=1}^{n}\left(\prod_{i=j+1}^{n} \frac{\lambda_{i}+\delta}{\lambda_{i}}\right) \frac{\alpha}{\lambda_{j}}} \\
\quad=\int_{0}^{u-b_{1}} V_{2 *}(u-x) p(x) d x+\int_{u-b_{1}}^{u} V_{1 *}(u-x) p(x) d x,
\end{gathered}
$$

当 $k=0,1,2, \ldots, n-1$ 时, 边界条件是

$$
\begin{aligned}
{\left.\left[\prod_{j=1}^{k}\left(1+\frac{\delta}{\lambda_{j}}-\frac{c-\alpha}{\lambda_{j}} \frac{\partial}{\partial u}\right)\right] \frac{\partial V_{2 *}(u)}{\partial u}\right|_{u=b_{2}-} } & =1 \\
{\left.\left[\prod_{j=1}^{k}\left(1+\frac{\delta}{\lambda_{j}}-\frac{c}{\lambda_{j}} \frac{\partial}{\partial u}\right)\right] V_{1 *}(u)\right|_{u=b_{1}-}=} & {\left.\left[\prod_{j=1}^{k}\left(1+\frac{\delta}{\lambda_{j}}-\frac{c-\alpha}{\lambda_{j}} \frac{\partial}{\partial u}\right)\right] V_{2 *}(u)\right|_{u=b_{1}+} } \\
& -\sum_{j=1}^{k}\left(\prod_{i=j+1}^{k} \frac{\lambda_{i}+\delta}{\lambda_{i}}\right) \frac{\alpha}{\lambda_{j}} .
\end{aligned}
$$

定义 $D$ 的矩母函数为

$$
M\left(u, z ; b_{1}, b_{2}\right)=E\left[\mathrm{e}^{z D} \mid U(0)=u\right]=E_{u}\left[\mathrm{e}^{z D}\right], \quad u \geqslant 0,
$$

其中 $z$ 为使得 $M\left(u, z ; b_{1}, b_{2}\right)$ 存在的实数. 与期望折现分红函数相似, 我们将 $M\left(u, z ; b_{1}, b_{2}\right)$ 写作

$$
M(u, z)=M\left(u, z ; b_{1}, b_{2}\right)= \begin{cases}M_{1}\left(u, z ; b_{1}, b_{2}\right)=M_{1}(u, z), & 0 \leqslant u<b_{1}, \\ M_{2}\left(u, z ; b_{1}, b_{2}\right)=M_{2}(u, z), & b_{1} \leqslant u \leqslant b_{2} .\end{cases}
$$

下面推导 $M(u, z)$ 满足积分 - 微分方程及其边界条件.

定理 $4 \quad M_{1}(u, z)$ 和 $M_{2}(u, z)$ 满足下面的积分 - 微分方程:

(1) 当 $0<u<b_{1}$ 时, 有

$$
\left[\prod_{j=1}^{n}\left(\frac{\delta z}{\lambda_{j}} \frac{\partial}{\partial z}-\frac{c}{\lambda_{j}} \frac{\partial}{\partial u}+1\right)\right] M_{1}(u, z)=\int_{0}^{u} M_{1}(u-x, z) p(x) d x+1-P(u) ;
$$

(2) 当 $b_{1}<u<b_{2}$ 时, 有

$$
\begin{aligned}
& {\left[\prod_{j=1}^{n}\left(\frac{\delta z}{\lambda_{j}} \frac{\partial}{\partial z}-\frac{c-\alpha}{\lambda_{j}} \frac{\partial}{\partial u}+1-\frac{\delta z}{\lambda_{j}}\right)\right] M_{2}(u, z)} \\
& \quad=\int_{0}^{u-b_{1}} M_{2}(u-x, z) p(x) d x+\int_{u-b_{1}}^{u} M_{1}(u-x, z) p(x) d x+1-P(u),
\end{aligned}
$$

当 $k=0,1,2, \ldots, n-1$ 时, 边界条件是

$$
\left.\left[\prod_{j=1}^{k}\left(\frac{\delta z}{\lambda_{j}} \frac{\partial}{\partial z}-\frac{c-\alpha}{\lambda_{j}} \frac{\partial}{\partial u}+1-\frac{\delta z}{\lambda_{j}}\right)\right] \frac{\partial M_{2}(u, z)}{\partial u}\right|_{u=b_{2}-}
$$




$$
\begin{array}{r}
\quad=\left.z\left[\prod_{j=1}^{k}\left(\frac{\delta z}{\lambda_{j}} \frac{\partial}{\partial z}-\frac{c-\alpha}{\lambda_{j}} \frac{\partial}{\partial u}+1-\frac{\delta z}{\lambda_{j}}\right)\right] M_{2}(u, z)\right|_{u=b_{2}-}, \\
{\left.\left[\prod_{j=1}^{k}\left(\frac{\delta z}{\lambda_{j}} \frac{\partial}{\partial z}-\frac{c}{\lambda_{j}} \frac{\partial}{\partial u}+1\right)\right] M_{1}(u, z)\right|_{u=b_{1}-}} \\
=\left.\left[\prod_{j=1}^{k}\left(\frac{\delta z}{\lambda_{j}} \frac{\partial}{\partial z}-\frac{c-\alpha}{\lambda_{j}} \frac{\partial}{\partial u}+1-\frac{\delta z}{\lambda_{j}}\right)\right] M_{2}(u, z)\right|_{u=b_{1}+} .
\end{array}
$$

证明 当 $0<u<b_{1}$ 时, 类似定理 1 的证明, 我们令 $M_{1 j}(u, z)$ 是风险过程在状态 $j(j=1, \ldots, n)$ 时的矩母函数, 显然有 $M_{1}(u)=M_{11}(u)$. 考虑在无穷小时间区间 $[0, d t]$ 内是否有索赔发生, 当 $j=1$, $\ldots, n-1$ 时, 我们有

$$
M_{1 j}(u, z)=\left(1-\lambda_{j} d t\right) M_{1 j}\left(u+c d t, z \mathrm{e}^{-\delta d t}\right)+\lambda_{j} d t M_{1 j+1}\left(u+c d t, z \mathrm{e}^{-\delta d t}\right),
$$

即

$$
M_{1 j+1}(u, z)=\left[\frac{z \delta}{\lambda_{j}} \frac{\partial}{\partial z}-\frac{c}{\lambda_{j}} \frac{\partial}{\partial u}+1\right] M_{1 j}(u, z)
$$

当 $j=n$ 时, 我们有

$$
\begin{aligned}
M_{1 n}(u)= & \left(1-\lambda_{n} d t\right) M_{1 n}\left(u+c d t, z \mathrm{e}^{-\delta d t}\right)+\lambda_{n} d t \int_{0}^{u+c d t} M_{1}\left(u+c d t-x, z \mathrm{e}^{-\delta d t}\right) p(x) d x \\
& +\lambda_{n} d t[1-P(u+c d t)]+o(d t) \\
= & M_{1 n}(u, z)-\lambda_{n} M_{1 n}(u, z) d t+c \frac{\partial M_{1 n}(u, z)}{\partial u} d t-z \delta d t \frac{\partial M_{1 n}(u, z)}{\partial z} \\
& +\lambda_{n} d t \int_{0}^{u+c d t} M_{1}(u-x, z) p(x) d x+\lambda_{n} d t[1-P(u+c d t)]+o(d t),
\end{aligned}
$$

整理得

$$
\left(\frac{\delta z}{\lambda_{n}} \frac{\partial}{\partial z}-\frac{c}{\lambda_{n}} \frac{\partial}{\partial u}+1\right) M_{1 n}(u)=\int_{0}^{u} M_{1}(u-x, z) p(x) d x+1-P(u) .
$$

利用 (3.28) 和 (3.29), 我们即得结论 (3.23).

(2) 当 $b_{1}<u<b_{2}$ 且 $j=1,2, \ldots, n-1$ 时, 我们有

$$
M_{2 j}(u, z)=\left(1-\lambda_{j} d t\right) \mathrm{e}^{z \alpha d t} M_{2 j}\left(u+(c-\alpha) d t, z \mathrm{e}^{-\delta d t}\right)+\lambda_{j} d t M_{2 j+1}\left(u+(c-\alpha) d t, z \mathrm{e}^{-\delta d t}\right),
$$

整理可得

$$
M_{2 j+1}(u, z)=\left[\frac{z \delta}{\lambda_{j}} \frac{\partial}{\partial z}-\frac{c-\alpha}{\lambda_{j}} \frac{\partial}{\partial u}+1-\frac{z \alpha}{\lambda_{j}}\right] M_{2 j}(u, z) .
$$

当 $j=n$ 时, 同理可得

$$
\begin{aligned}
\left(\frac{\delta z}{\lambda_{n}} \frac{\partial}{\partial z}-\frac{c-\alpha}{\lambda_{n}} \frac{\partial}{\partial u}+1-\frac{z \alpha}{\lambda_{n}}\right) M_{1 n}(u)= & \int_{0}^{u-b_{1}} M_{2}(u-x, z) p(x) d x \\
& +\int_{u-b_{1}}^{u} M_{1}(u-x, z) p(x) d x+1-P(u) .
\end{aligned}
$$


利用 (3.31) 和 (3.32) 我们即得结论 (3.20).

下面证明边界条件 (3.25). 当 $u=b_{2}$ 且 $j=1,2, \ldots, n-1$ 时, 类似可得

$$
M_{2 j}\left(b_{2}, z\right)=\left(1-\lambda_{j} d t\right) \mathrm{e}^{z \alpha d t} \mathrm{e}^{z(c-\alpha) d t} M_{2 j}\left(b_{2}, z \mathrm{e}^{-\delta d t}\right)+\lambda_{j} d t \mathrm{e}^{z(c-\alpha) d t} \mathrm{e}^{z \alpha d t} M_{2 j+1}\left(b_{2}, z \mathrm{e}^{-\delta d t}\right) .
$$

利用 Taylor 展式有

$$
M_{2 j+1}\left(b_{2}, z\right)=\frac{z \delta}{\lambda_{j}} \frac{\partial M_{2 j}\left(b_{2}, z\right)}{\partial z}-\left(\frac{z c}{\lambda_{j}}-1\right) M_{2 j}\left(b_{2}, z\right) .
$$

又在 $(3.31)$ 中令 $u \uparrow b_{2}$,

$$
M_{2 j+1}\left(b_{2}, z\right)=\frac{z \delta}{\lambda_{j}} \frac{\partial M_{2 j}\left(b_{2}, z\right)}{\partial z}-\frac{c-\alpha}{\lambda_{j}} \frac{\partial M_{2 j}\left(b_{2}, z\right)}{\partial u}+1-\frac{z \alpha}{\lambda_{j}} M_{2 j}\left(b_{2}, z\right) .
$$

把上两式作比较可得

$$
\left.\frac{\partial M_{2 j}(u, z)}{\partial u}\right|_{u=b_{2}-}=\left.z M_{2 j}(u, z)\right|_{u=b_{2}-}, \quad j=1,2, \ldots, n-1 .
$$

由上式可得条件 $(3.25)$ 成立. 利用公式 $M_{1}\left(b_{1}, z\right)=M_{2}\left(b_{1}, z\right)$ 及 $(3.28)$ 和 (3.31) 可得 (3.26) 成立. 定 理得证.

例 1 若 $n=2$ 且 $p(x)=\beta \mathrm{e}^{-\beta x}, \beta>0, x \geqslant 0$, 我们有

$$
\left[\prod_{j=1}^{2}\left(\frac{c}{\lambda_{j}} \frac{\partial}{\partial u}-\left(1+\frac{\delta}{\lambda_{j}}\right)\right)\right] V_{1 *}(u)=\beta \mathrm{e}^{\beta u} \int_{0}^{u} V_{1 *}(x) \mathrm{e}^{-\beta x} d x, \quad 0<u<b_{1},
$$

且当 $b_{1}<u<b_{2}$ 时, 有

$$
\begin{aligned}
& {\left[\prod_{j=1}^{2}\left(1+\frac{\delta}{\lambda_{j}}-\frac{c-\alpha}{\lambda_{j}} \frac{\partial}{\partial u}\right)\right] V_{2 *}(u)-\frac{\lambda_{2}+\delta}{\lambda_{2}} \frac{\alpha}{\lambda_{1}}-\frac{\alpha}{\lambda_{1}}} \\
& \quad=\beta \mathrm{e}^{-\beta u} \int_{0}^{b_{1}} V_{1 *}(x) \mathrm{e}^{-\beta x} d x+\beta \mathrm{e}^{-\beta u} \int_{b_{1}}^{u} V_{2 *}(x) \mathrm{e}^{-\beta x} d x .
\end{aligned}
$$

在 (3.33) 两端同时作用算子 $\frac{\partial}{\partial u}+\beta$ 得

$$
\left(\frac{\partial}{\partial u}+\beta\right) \prod_{j=1}^{2}\left(\frac{c}{\lambda_{j}} \frac{\partial}{\partial u}-\left(1+\frac{\delta}{\lambda_{j}}\right)\right) V_{1 *}(u)=\beta V_{1 *}(u) .
$$

由上式可知其特征方程为

$$
\frac{\left(c r-\lambda_{1}-\delta\right)\left(c r-\lambda_{2}-\delta\right)}{\lambda_{1} \lambda_{2}}=\frac{\beta}{\beta+r} .
$$

通过上式两边的函数图象可知, 上式的特征方程有且只有三个实根 $r_{1}, r_{2}$ 和 $r_{3}$ 满足 $-\beta<r_{1}<0<r_{2}$ $<r_{3}$. 故有

$$
V_{1 *}(u)=c_{1} \mathrm{e}^{r_{1} u}+c_{2} \mathrm{e}^{r_{2} u}+c_{3} \mathrm{e}^{r_{3} u},
$$

其中 $c_{1}, c_{2}$ 和 $c_{3}$ 是常数. 在 (3.34) 两端同时作用算子 $\frac{\partial}{\partial u}+\beta$, 我们有

$$
(c-\alpha)^{2} \frac{\partial^{3} V_{2 *}(u)}{\partial u^{3}}+(c-\alpha)\left[\beta(c-\alpha)-\left(\lambda_{1}+\lambda_{2}+2 \delta\right)\right] \frac{\partial^{2} V_{2 *}(u)}{\partial u^{2}}
$$




$$
\begin{aligned}
& +\left[\left(\lambda_{1}+\delta\right)\left(\lambda_{2}+\delta\right)-\beta(c-\alpha)\left(\lambda_{1}+\lambda_{2}+2 \delta\right)\right] \frac{\partial V_{2 *}(u)}{\partial u} \\
& +\beta \delta\left(\lambda_{1}+\lambda_{2}+\delta\right) V_{2 *}(u)-\alpha \beta\left(\lambda_{1}+\lambda_{2}+\delta\right)=0,
\end{aligned}
$$

易知, (3.36) 的一个解为 $\frac{\alpha}{\delta}$. 故 (3.36) 的一个解可以表示为

$$
V_{2 *}(u)=\frac{\alpha}{\delta}+\widehat{c}_{1} \mathrm{e}^{\widehat{r}_{1} u}+\widehat{c}_{2} \mathrm{e}^{\widehat{r}_{2} u}+\widehat{c}_{3} \mathrm{e}^{\widehat{r}_{3} u},
$$

其中 $\widehat{c}_{1}, \widehat{c}_{2}$ 和 $\widehat{c}_{3}$ 是待定系数. 又由 (3.21) 和 (3.22), 我们得

$$
\begin{aligned}
& \widehat{c}_{1} \widehat{r}_{1} \mathrm{e}^{\widehat{\mathrm{T}}_{1} b_{2}}+\widehat{c}_{2} \widehat{r}_{2} \mathrm{e}^{\widehat{r}_{2} b_{2}}+\widehat{c}_{3} \widehat{r}_{3} \mathrm{e}^{\widehat{r}_{3} b_{2}}=1, \\
& \widehat{c}_{1} \widehat{r}_{1}^{2} \mathrm{e}^{\widehat{r}_{1} b_{2}}+\widehat{c}_{2} \widehat{r}_{2}^{2} \mathrm{e}^{\widehat{r}_{2} b_{2}}+\widehat{c}_{3} \widehat{r}_{3}^{2} \mathrm{e}^{\widehat{r}_{3} b_{2}}=\frac{\delta}{c-\alpha}, \\
& c_{1} \mathrm{e}^{r_{1} b_{1}}+c_{2} \mathrm{e}^{r_{2} b_{1}}+c_{3} \mathrm{e}^{r_{3} b_{1}}-\widehat{c}_{1} \widehat{r}_{1} \mathrm{e}^{\widehat{r}_{1} b_{1}}-\widehat{c}_{2} \widehat{r}_{2} \mathrm{e}^{\widehat{r}_{2} b_{1}}-\widehat{c}_{3} \widehat{r}_{3} \mathrm{e}^{\widehat{r}_{3} b_{1}}=\frac{\alpha}{\delta}, \\
& c_{1} c r_{1} \mathrm{e}^{r_{1} b_{1}}+c_{2} c r_{2} \mathrm{e}^{r_{2} b_{1}}+c_{3} c r_{3} \mathrm{e}^{r_{3} b_{1}}-(c-\alpha)\left(\widehat{c}_{1} \widehat{r}_{1} \mathrm{e}^{\widehat{r}_{1} b_{1}}-\widehat{c}_{2} \widehat{r}_{2} \mathrm{e}^{\widehat{r}_{2} b_{1}}-\widehat{c}_{3} \widehat{r}_{3} \mathrm{e}^{\widehat{r}_{3} b_{1}}\right)=\alpha .
\end{aligned}
$$

把 (3.35) 和 (3.37) 分别带入到 (3.33) 和 (3.34) 中, 又利用此方程对所有的满足 $0 \leqslant u \leqslant b_{1}$ 和 $b_{1} \leqslant u$ $\leqslant b_{2}$ 的 $u$ 都成立, 故 $\mathrm{e}^{-\beta u}$ 前的系数必须为零. 故

$$
\begin{aligned}
& \frac{c_{1}}{r_{1}+\beta}+\frac{c_{2}}{r_{2}+\beta}+\frac{c_{3}}{r_{3}+\beta}=0, \\
& \frac{c_{1}}{r_{1}+\beta} \mathrm{e}^{r_{1} b_{1}}+\frac{c_{2}}{r_{2}+\beta} \mathrm{e}^{r_{2} b_{1}}+\frac{c_{3}}{r_{3}+\beta} \mathrm{e}^{r_{3} b_{1}}=\frac{\alpha}{\delta \beta}+\frac{\widehat{c}_{1}}{\widehat{r}_{1}+\beta} \mathrm{e}^{\widehat{r}_{1} b_{1}}+\frac{\widehat{c}_{2}}{\widehat{r}_{2}+\beta} \mathrm{e}^{\widehat{r}_{2} b_{1}}+\frac{\widehat{c}_{3}}{\widehat{r}_{3}+\beta} \mathrm{e}^{\widehat{r}_{3} b_{1}} .
\end{aligned}
$$

利用 (3.38)-(3.43), 我们得系数 $c_{1}, c_{2}, c_{3}, \widehat{c_{1}}, \widehat{c_{2}}$ 和 $\widehat{c_{3}}$ 的表达式

$$
c_{1}=\frac{\triangle_{1}}{\triangle}, \quad c_{2}=\frac{\triangle_{2}}{\triangle}, \quad c_{3}=\frac{\triangle_{3}}{\triangle}, \quad \widehat{c}_{1}=\frac{\triangle_{4}}{\triangle}, \quad \widehat{c}_{2}=\frac{\triangle_{5}}{\triangle}, \quad \widehat{c}_{3}=\frac{\triangle_{6}}{\triangle},
$$

其中

$$
\begin{aligned}
& \triangle=\left|\begin{array}{cccccc}
\frac{1}{r_{1}+\beta} & \frac{1}{r_{2}+\beta} & \frac{1}{r_{3}+\beta} & 0 & 0 & 0 \\
\frac{\mathrm{e}^{r_{1} b_{1}}}{r_{1}+\beta} & \frac{\mathrm{e}^{r_{2} b_{1}}}{r_{2}+\beta} & \frac{\mathrm{e}^{r_{3} b_{1}}}{r_{3}+\beta} & -\frac{\mathrm{e}^{\widehat{r}_{1} b_{1}}}{\widehat{r}_{1}+\beta} & -\frac{\mathrm{e}^{\widehat{r}_{2} b_{1}}}{\widehat{r}_{2}+\beta} & -\frac{\mathrm{e}^{\widehat{r}_{3} b_{1}}}{\widehat{r}_{3}+\beta} \\
0 & 0 & 0 & \widehat{r}_{1} \mathrm{e}^{\widehat{r}_{1} b_{2}} & \widehat{r}_{2} \mathrm{e}^{\widehat{r}_{2} b_{2}} & \widehat{r}_{3} \mathrm{e}^{\widehat{r}_{3} b_{2}} \\
0 & 0 & 0 & \widehat{r}_{1}^{2} \mathrm{e}^{\widehat{r}_{1} b_{2}} & \widehat{r}_{2}^{2} \mathrm{e}^{\widehat{r}_{2} b_{2}} & \widehat{r}_{3}^{2} \mathrm{e}^{\widehat{r}_{3} b_{2}} \\
\mathrm{e}^{r_{1} b_{1}} & \mathrm{e}^{r_{2} b_{1}} & \mathrm{e}^{r_{3} b_{1}} & -\widehat{r}_{1} \mathrm{e}^{\widehat{r}_{1} b_{1}} & -\widehat{r}_{2} \mathrm{e}^{\widehat{r}_{2} b_{1}} & -\widehat{r}_{3} \mathrm{e}^{\widehat{r}_{3} b_{1}} \\
c r_{1} \mathrm{e}^{r_{1} b_{1}} & c r_{2} \mathrm{e}^{r_{2} b_{1}} & c r_{3} \mathrm{e}^{r_{3} b_{1}} & -(c-\alpha) \widehat{r}_{1} \mathrm{e}^{\widehat{r}_{1} b_{1}} & -(c-\alpha) \widehat{r}_{2} \mathrm{e}^{\widehat{r}_{2} b_{1}} & -(c-\alpha) \widehat{r}_{3} \mathrm{e}^{\widehat{\mathrm{r}}_{3} b_{1}}
\end{array}\right|, \\
& \triangle_{1}=\left|\begin{array}{cccccc}
0 & \frac{1}{r_{3}+\beta} & 0 & 0 & 0 & 0 \\
\frac{\alpha}{\delta \beta} & \frac{\mathrm{e}^{r_{2} b_{1}}}{r_{2}+\beta} & \frac{\mathrm{e}^{r_{3} b_{1}}}{r_{3}+\beta} & -\frac{\mathrm{e}^{\widehat{r}_{1} b_{1}}}{\widehat{r}_{1}+\beta} & -\frac{\mathrm{e}^{\widehat{r}_{2} b_{1}}}{\widehat{r}_{2}+\beta} & -\frac{\mathrm{e}^{\widehat{r}_{3} b_{1}}}{\widehat{r}_{3}+\beta} \\
1 & 0 & 0 & \widehat{r}_{1} \mathrm{e}^{\widehat{r}_{1} b_{2}} & \widehat{r}_{2} \mathrm{e}^{\widehat{r}_{2} b_{2}} & \widehat{r}_{3} \mathrm{e}^{\widehat{r}_{3} b_{2}} \\
\frac{\delta}{c-\alpha} & 0 & 0 & \widehat{r}_{1}^{2} \mathrm{e}^{\widehat{r}_{1} b_{2}} & \widehat{r}_{2}^{2} \mathrm{e}^{\widehat{r}_{2} b_{2}} & \widehat{r}_{3}^{2} \mathrm{e}^{\widehat{r}_{3} b_{2}} \\
\frac{\alpha}{\delta} & \mathrm{e}^{r_{2} b_{1}} & \mathrm{e}^{r_{3} b_{1}} & -\widehat{r}_{1} \mathrm{e}^{\widehat{r}_{1} b_{1}} & -\widehat{r}_{2} \mathrm{e}^{\widehat{r}_{2} b_{1}} & -\widehat{r}_{3} \mathrm{e}^{\widehat{r}_{3} b_{1}} \\
\alpha & c r_{2} \mathrm{e}^{r_{2} b_{1}} & c r_{3} \mathrm{e}^{r_{3} b_{1}} & -(c-\alpha) \widehat{r}_{1} \mathrm{e}^{\widehat{r}_{1} b_{1}} & -(c-\alpha) \widehat{r}_{2} \mathrm{e}^{\widehat{r}_{2} b_{1}} & -(c-\alpha) \widehat{r}_{3} \mathrm{e}^{\widehat{r}_{3} b_{1}}
\end{array}\right|,
\end{aligned}
$$




$$
\begin{aligned}
& \triangle_{2}=\left|\begin{array}{cccccc}
\frac{1}{r_{1}+\beta} & 0 & \frac{1}{r_{3}+\beta} & 0 & 0 & 0 \\
\frac{\mathrm{e}^{r_{1} b_{1}}}{r_{1}+\beta} & \frac{\alpha}{\delta \beta} & \frac{\mathrm{e}^{r_{3} b_{1}}}{r_{3}+\beta} & -\frac{\mathrm{e}^{\widehat{r}_{1} b_{1}}}{\widehat{r}_{1}+\beta} & -\frac{\mathrm{e}^{\widehat{r}_{2} b_{1}}}{\widehat{r}_{2}+\beta} & -\frac{\mathrm{e}^{\widehat{r}_{3} b_{1}}}{\widehat{r}_{3}+\beta} \\
0 & 1 & 0 & \widehat{r}_{1} \mathrm{e}^{\widehat{r}_{1} b_{2}} & \widehat{r}_{2} \mathrm{e}^{\widehat{r}_{2} b_{2}} & \widehat{r}_{3} \mathrm{e}^{\widehat{r}_{3} b_{2}} \\
0 & \frac{\delta}{c-\alpha} & 0 & \widehat{r}_{1}^{2} \mathrm{e}^{\widehat{r}_{1} b_{2}} & \widehat{r}_{2}^{2} \mathrm{e}^{\widehat{r}_{2} b_{2}} & \widehat{r}_{3}^{2} \mathrm{e}^{\widehat{r}_{3} b_{2}} \\
\mathrm{e}^{r_{1} b_{1}} & \frac{\alpha}{\delta} & \mathrm{e}^{r_{3} b_{1}} & -\widehat{r}_{1} \mathrm{e}^{\widehat{r}_{1} b_{1}} & -\widehat{r}_{2} \mathrm{e}^{\widehat{r}_{2} b_{1}} & -\widehat{r}_{3} \mathrm{e}^{\widehat{C}_{3} b_{1}} \\
c r_{1} \mathrm{e}^{r_{1} b_{1}} & \alpha & c r_{3} \mathrm{e}^{r_{3} b_{1}} & -(c-\alpha) \widehat{r}_{1} \mathrm{e}^{\widehat{r}_{1} b_{1}} & -(c-\alpha) \widehat{r}_{2} \mathrm{e}^{\widehat{r}_{2} b_{1}} & -(c-\alpha) \widehat{r}_{3} \mathrm{e}^{\widehat{r}_{3} b_{1}}
\end{array}\right|, \\
& \triangle_{3}=\left|\begin{array}{cccccc}
\frac{1}{r_{1}+\beta} & \frac{1}{r_{2}+\beta} & 0 & 0 & 0 & 0 \\
\frac{\mathrm{e}^{r_{1} b_{1}}}{r_{1}+\beta} & \frac{\mathrm{e}^{r_{2} b_{1}}}{r_{2}+\beta} & \frac{\alpha}{\delta \beta} & -\frac{\mathrm{e}^{\widehat{r}_{1} b_{1}}}{\widehat{r}_{1}+\beta} & -\frac{\mathrm{e}^{\widehat{r}_{2} b_{1}}}{\widehat{r}_{2}+\beta} & -\frac{\mathrm{e}^{\widehat{r}_{3} b_{1}}}{\widehat{r}_{3}+\beta} \\
0 & 0 & 1 & \widehat{r}_{1} \mathrm{e}^{\widehat{r}_{1} b_{2}} & \widehat{r}_{2} \mathrm{e}^{\widehat{r}_{2} b_{2}} & \widehat{r}_{3} \mathrm{e}^{\widehat{r}_{3} b_{2}} \\
0 & 0 & \frac{\delta}{c-\alpha} & \widehat{r}_{1}^{2} \mathrm{e}^{\widehat{r}_{1} b_{2}} & \widehat{r}_{2}^{2} \mathrm{e}^{\widehat{r}_{2} b_{2}} & \widehat{r}_{3}^{2} \mathrm{e}^{\widehat{r}_{3} b_{2}} \\
\mathrm{e}^{r_{1} b_{1}} & \mathrm{e}^{r_{2} b_{1}} & \frac{\alpha}{\delta} & -\widehat{r}_{1} \mathrm{e}^{\widehat{r}_{1} b_{1}} & -\widehat{r}_{2} \mathrm{e}^{\widehat{r}_{2} b_{1}} & -\widehat{r}_{3} \mathrm{e}^{\widehat{r}_{3} b_{1}} \\
c r_{1} \mathrm{e}^{r_{1} b_{1}} & c r_{2} \mathrm{e}^{r_{2} b_{1}} & \alpha & -(c-\alpha) \widehat{r}_{1} \mathrm{e}^{\widehat{r}_{1} b_{1}} & -(c-\alpha) \widehat{r}_{2} \mathrm{e}^{\widehat{r}_{2} b_{1}} & -(c-\alpha) \widehat{r}_{3} \mathrm{e}^{\widehat{r}_{3} b_{1}}
\end{array}\right|, \\
& \triangle_{4}=\left|\begin{array}{cccccc}
\frac{1}{r_{1}+\beta} & \frac{1}{r_{2}+\beta} & \frac{1}{r_{3}+\beta} & 0 & 0 & 0 \\
\frac{\mathrm{e}^{r_{1} b_{1}}}{r_{1}+\beta} & \frac{\mathrm{e}^{r_{2} b_{1}}}{r_{2}+\beta} & \frac{\mathrm{e}^{r_{3} b_{1}}}{r_{3}+\beta} & \frac{\alpha}{\delta \beta} & -\frac{\mathrm{e}^{\widehat{r}_{2} b_{1}}}{\widehat{r}_{2}+\beta} & -\frac{\mathrm{e}^{\widehat{r}_{3} b_{1}}}{\widehat{r}_{3}+\beta} \\
0 & 0 & 0 & 1 & \widehat{r}_{2} \mathrm{e}^{\widehat{r}_{2} b_{2}} & \widehat{r}_{3} \mathrm{e}^{\widehat{r}_{3} b_{2}} \\
0 & 0 & 0 & \frac{\delta}{c-\alpha} & \widehat{r}_{2}^{2} \mathrm{e}^{\widehat{r}_{2} b_{2}} & \widehat{r}_{3}^{2} \mathrm{e}^{\widehat{r}_{3} b_{2}} \\
\mathrm{e}^{r_{1} b_{1}} & \mathrm{e}^{r_{2} b_{1}} & \mathrm{e}^{r_{3} b_{1}} & \frac{\alpha}{\delta} & -\widehat{r}_{2} \mathrm{e}^{\widehat{r}_{2} b_{1}} & -\widehat{r}_{3} \mathrm{e}^{\widehat{r}_{3} b_{1}} \\
c r_{1} \mathrm{e}^{r_{1} b_{1}} & c r_{2} \mathrm{e}^{r_{2} b_{1}} & c r_{3} \mathrm{e}^{r_{3} b_{1}} & \alpha & -(c-\alpha) \widehat{r}_{2} \mathrm{e}^{\widehat{r}_{2} b_{1}} & -(c-\alpha) \widehat{r}_{3} \mathrm{e}^{\widehat{r}_{3} b_{1}}
\end{array}\right|, \\
& \triangle_{5}=\left|\begin{array}{cccccc}
\frac{1}{r_{1}+\beta} & \frac{1}{r_{2}+\beta} & \frac{1}{r_{3}+\beta} & 0 & 0 & 0 \\
\frac{\mathrm{e}^{r_{1} b_{1}}}{r_{1}+\beta} & \frac{\mathrm{e}^{r_{2} b_{1}}}{r_{2}+\beta} & \frac{\mathrm{e}^{r_{3} b_{1}}}{r_{3}+\beta} & -\frac{\mathrm{e}^{\widehat{r}_{1} b_{1}}}{\widehat{r}_{1}+\beta} & \frac{\alpha}{\delta \beta} & -\frac{\mathrm{e}^{\widehat{r}_{3} b_{1}}}{\widehat{r}_{3}+\beta} \\
0 & 0 & 0 & \widehat{r}_{1} \mathrm{e}^{\widehat{r}_{1} b_{2}} & 1 & \widehat{r}_{3} \mathrm{e}^{\widehat{r}_{3} b_{2}} \\
0 & 0 & 0 & \widehat{r}_{1}^{2} \mathrm{e}^{\widehat{r}_{1} b_{2}} & \frac{\delta}{c-\alpha} & \widehat{r}_{3}^{2} \mathrm{e}^{\widehat{r}_{3} b_{2}} \\
\mathrm{e}^{r_{1} b_{1}} & \mathrm{e}^{r_{2} b_{1}} & \mathrm{e}^{r_{3} b_{1}} & -\widehat{r}_{1} \mathrm{e}^{\widehat{r}_{1} b_{1}} & \frac{\alpha}{\delta} & -\widehat{r}_{3} \mathrm{e}^{\widehat{r}_{3} b_{1}} \\
c r_{1} \mathrm{e}^{r_{1} b_{1}} & c r_{2} \mathrm{e}^{r_{2} b_{1}} & c r_{3} \mathrm{e}^{r_{3} b_{1}} & -(c-\alpha) \widehat{r}_{1} \mathrm{e}^{\widehat{r}_{1} b_{1}} & \alpha & -(c-\alpha) \widehat{r}_{3} \mathrm{e}^{\widehat{r}_{3} b_{1}}
\end{array}\right|, \\
& \triangle_{6}=\left|\begin{array}{cccccc}
\frac{1}{r_{1}+\beta} & \frac{1}{r_{2}+\beta} & \frac{1}{r_{3}+\beta} & 0 & 0 & 0 \\
\frac{\mathrm{e}^{r_{1} b_{1}}}{r_{1}+\beta} & \frac{\mathrm{e}^{r_{2} b_{1}}}{r_{2}+\beta} & \frac{\mathrm{e}^{r_{3} b_{1}}}{r_{3}+\beta} & -\frac{\mathrm{e}^{\widehat{r}_{1} b_{1}}}{\widehat{r}_{1}+\beta} & -\frac{\mathrm{e}^{\widehat{r}_{2} b_{1}}}{\widehat{r}_{2}+\beta} & \frac{\alpha}{\delta \beta} \\
0 & 0 & 0 & \widehat{r}_{1} \mathrm{e}^{\widehat{r}_{1} b_{2}} & \widehat{r}_{2} \mathrm{e}^{\widehat{r}_{2} b_{2}} & 1 \\
0 & 0 & 0 & \widehat{r}_{1}^{2} \mathrm{e}^{\widehat{r}_{1} b_{2}} & \widehat{r}_{2}^{2} \mathrm{e}^{\widehat{r}_{2} b_{2}} & \frac{\delta}{c-\alpha} \\
\mathrm{e}^{r_{1} b_{1}} & \mathrm{e}^{r_{2} b_{1}} & \mathrm{e}^{r_{3} b_{1}} & -\widehat{r}_{1} \mathrm{e}^{\widehat{r}_{1} b_{1}} & -\widehat{r}_{2} \mathrm{e}^{\widehat{r}_{2} b_{1}} & \frac{\alpha}{\delta} \\
c r_{1} \mathrm{e}^{r_{1} b_{1}} & c r_{2} \mathrm{e}^{r_{2} b_{1}} & c r_{3} \mathrm{e}^{r_{3} b_{1}} & -(c-\alpha) \widehat{r}_{1} \mathrm{e}^{\widehat{r}_{1} b_{1}} & -(c-\alpha) \widehat{r}_{2} \mathrm{e}^{\widehat{r}_{2} b_{1}} & \alpha
\end{array}\right| .
\end{aligned}
$$

致谢 作者衰心的感谢审稿人对本文提出的宝贵修改意见. 


\section{参考文献}

1 Lin X, Willmot G E, Drekic S. The classical risk model with a constant dividend barrier: Analysis of the Gerber-Shiu discounted penalty function. Insurance Math Econom, 2003, 33: 551-566

2 Yuen K C, Yin C. On optimality of the barrier strategy for a general Lévy risk process. Math Comput Modelling, 2011, 53: $1700-1707$

3 Jeanblanc-Picqué M, Shiryaev A N. Optimization of the flow of dividends. Russian Math Surveys, 1995, 50: 257-277

4 Asmussen S, Taksar M. Controlled diffusion models for optimal dividend pay-out. Insurance Math Econom, 1997, 20: $1-15$

5 Ng A C Y. On a dual model with a dividend threshold. Insurance Math Econom, 2009, 44: 315-324

6 Wan N. Dividend payments with a threshold strategy in the compound Poisson risk model perturbed by diffusion. Insurance Math Econom, 2007, 40: 509-523

7 Albrecher H, Badescu A L, Landriault D. On the dual risk model with tax payments. Insurance Math Econom, 2008, 42: $1086-1094$

8 Gerber H U, Shiu E S W. On the time value of ruin. N Am Actuar J, 1998, 2: 48-78

9 Fang Y, Wu R. Optimal dividends in the Brownian motion risk model with interest. J Comput Appl Math, 2009, 229: $145-151$

10 Lin X, Pavlova K P. The compound Poisson risk model with a threshold dividend strategy. Insurance Math Econom, 2006, 38: $57-80$

11 孟辉. 方差保费准则下的最优脉冲控制. 中国科学: 数学, 2013, 43: 925-939

12 Yin C, Wen Y. An extension of Paulsen-Gjessing's risk model with stochastic return on investments. Insurance Math Econom, 2013, 52: 469-476

13 Zhang Z, Yang H, Li S. The perturbed compound Poisson risk model with two-sided jumps. J Comput Appl Math, 2010, 233: $1773-1784$

$14 \mathrm{Ng} \mathrm{A} \mathrm{C} \mathrm{Y.} \mathrm{On} \mathrm{the} \mathrm{upcrossing} \mathrm{and} \mathrm{downcrossing} \mathrm{probabilities} \mathrm{of} \mathrm{a} \mathrm{dual} \mathrm{risk} \mathrm{model} \mathrm{with} \mathrm{phase-type} \mathrm{gains.} \mathrm{Astin} \mathrm{Bull,}$ 2010, 40: 281-306

15 Grandell J. Aspects of Risk Theory. New York: Springer-Verlag, 1991

16 Albrecher H, Claramunt M M, Mármol M. On the distribution of dividend payments in a Sparre Andersen model with generalized Erlang $(n)$ interclaim times. Insurance Math Econom, 2005, 37: 324-334

17 Gerber H U, Shiu E S W. On optimal dividend strategy in the compound Poisson model. N Am Actuar J, 2006, 10: $76-93$

\section{A generalized $\operatorname{Erlang}(n)$ risk model with a hybrid dividend strategy}

\section{WEN YuZhen \& YIN ChuanCun}

Abstract In this paper, we consider the generalized $\operatorname{Erlang}(n)$ risk model with a hybrid dividend strategy. Using the lack-of-memory property of the exponential distribution, we derive integro-differential equations with boundary conditions satisfied by the expectation of the sum of discounted dividends until ruin and the momentgenerating function of the discounted dividend payments until ruin respectively. Explicit solutions of the expectation of the discounted dividend are given for a generalized Erlang(2) risk model and exponential-distributed claim amounts.

Keywords hybrid dividend strategy, discounted dividend payments, generalized Erlang $(n)$ distribution MSC(2010) 62P05, 62K05

doi: $10.1360 / 012014-46$ 